\title{
Screening of a Large Cohort of Leber Congenital Amaurosis and Retinitis Pigmentosa Patients Identifies Novel LCA5 Mutations and New Genotype-Phenotype Correlations
}

Donna S. Mackay, ${ }_{1}^{1 \dagger}$ Arundhati Dev Borman, ${ }^{1,2 \dagger}$ Ruifang Sui, ${ }^{3}$ L. Ingeborgh van den Born, ${ }^{4}$ Eliot L. Berson, ${ }^{5}$ Louise A. Ocaka, Alice E. Davidson, ${ }^{1}$ John R. Heckenlively, ${ }^{6}$ Kari Branham, ${ }^{6}$ Huanan Ren, ${ }^{7}$ Irma Lopez, ${ }^{7}$ Maleeha Maria, ${ }^{8,9}$ Maleeha Azam, ${ }^{8,9}$ Arjen Henkes, ${ }^{8}$ Ellen Blokland, ${ }^{8}$ [ LCA5 Study Group (see Acknowledgments for Universities), Sten Andreasson, Elfride de Baere, Jean Bennett, Gerald J. Chader, Wolfgang Berger, Irina Golovleva, Jacquie Greenberg, Anneke I. den Hollander, Caroline C.W. Klaver, B. Jeroen Klevering, Birgit Lorenz, Markus N. Preising, Raj Ramesar, Lisa Roberts, Ronald Roepman, Klaus Rohrschneider, Bernd Wissinger], Raheel Qamar, ${ }^{9,10}$ Andrew R. Webster, ${ }^{1,2}$ Frans P.M. Cremers, ${ }^{8 \ddagger}$ Anthony T. Moore, ${ }^{1,2 \ddagger}$ and Robert K. Koenekoop ${ }^{7 * \ddagger}$

${ }^{1}$ Department of Human Genetics, Institute of Ophthalmology, UCL, London, UK; ${ }^{2}$ Moorfields Eye Hospital, London, UK; ${ }^{3}$ Ophthalmology, Peking Union Medical College Hospital, Beijing, China; ${ }^{4}$ The Rotterdam Eye Hospital, Rotterdam, The Netherlands, ${ }^{5}$ Berman-Gund Laboratory for the Study of Retinal Degenerations, Harvard Medical School, Massachusetts Eye and Ear, Boston, Massachusetts; ${ }^{6}$ Kellogg Eye Center, Department of Ophthalmology and Visual Sciences, University of Michigan Medical School, Ann Arbor, Michigan; ${ }^{7}$ McGill Ocular Genetics Laboratory, Departments of Pediatric Surgery, Human Genetics and Ophthalmology, McGill University Health Centre, Montreal, Quebec, Canada; ${ }^{8}$ Department of Human Genetics, Radboud University Medical Centre, and Nijmegen Centre for Molecular Life Sciences, Nijmegen, The Netherlands; ${ }^{9}$ Department of Biosciences, COMSATS Institute of Information Technology, Islamabad, Pakistan; ${ }^{10}$ Al-Nafees Medical College \& Hospital, Isra University, Islamabad, Pakistan

Communicated by Daniel F. Schorderet

Received 29 March 2013; accepted revised manuscript 5 August 2013.

Published online 14 August 2013 in Wiley Online Library (www.wiley.com/humanmutation). DOI: 10.1002/humu.22398

\begin{abstract}
This study was undertaken to investigate the prevalence of sequence variants in LCA5 in patients with Leber congenital amaurosis (LCA), early-onset retinal dystrophy (EORD), and autosomal recessive retinitis pigmentosa (arRP); to delineate the ocular phenotypes; and to provide an overview of all published LCA5 variants in an online database. Patients underwent standard ophthalmic evaluations after providing informed consent. In selected patients, optical coherence tomography (OCT) and fundus autofluorescence imaging were possible. DNA samples from 797 unrelated patients with LCA and 211 with the various types of retinitis pigmentosa (RP) were screened by Sanger sequence analysis of all LCA5 exons and intron/exon junctions. Some LCA patients were pre-
\end{abstract}

[This article was originally published online on 17 September 2013 and appeared in the issue (vol. 34, iss. 11) on 9 October 2013. Subsequently, an error in an author's name was identified and corrected on 150 ctober 2013.]

†These authors contributed equally to this study.

$¥$ These senior authors contributed equally to this study.

*Correspondence to: Robert K. Koenekoop, McGill University Health Centre, McGill Ocular Genetics Centre, 2300 Tupper, Montreal, Quebec H3H 1P3, Canada. E-mail: robkoenekoop@hotmail.com

Contract grant sponsor: Fight for Sight (A.R.W., A.T.M.), National Institute for Health Research UK- Moorfields Eye Hospital Biomedical Research Centre (A.R.W., A.T.M.), Foundation Fighting Blindness-USA (A.R.W., A.T.M.), Ulvercroft Foundation (A.R.W., A.T.M.), Foundation for Retinal Research (F.P.M.C., R.K.K., A.I.d.H., J.B., R.R.), Foundation Fighting Blindness-Canada (R.K.K.), CIHR (R.K.K.), NIH (R.K.K.), Reseau Vision (R.K.K.), FRSO (R.K.K.), Retina South Africa (J.G., R.R.). screened by APEX technology or selected based on homozygosity mapping. In silico analyses were performed to assess the pathogenicity of the variants. Segregation analysis was performed where possible. Published and novel LCA5 variants were collected, amended for their correct nomenclature, and listed in a Leiden Open Variation Database (LOVD). Sequence analysis identified 18 new probands with 19 different LCA5 variants. Seventeen of the 19 LCA5 variants were novel. Except for two missense variants and one splice site variant, all variants were protein-truncating mutations. Most patients expressed a severe phenotype, typical of LCA. However, some LCA subjects had better vision and intact inner segment/outer segment (IS/OS) junctions on OCT imaging. In two families with LCA5 variants, the phenotype was more compatible with EORD with affected individuals displaying preserved islands of retinal pigment epithelium. One of the families with a milder phenotype harbored a homozygous splice site mutation; a second family was found to have a combination of a stop mutation and a missense mutation. This is the largest LCA5 study to date. We sequenced 1,008 patients ( 797 with LCA, 211 with arRP) and identified 18 probands with LCA5 mutations. Mutations in LCA5 are a rare cause of childhood retinal dystrophy accounting for $\sim 2 \%$ of disease in this cohort, and the majority of LCA5 mutations are likely null. The LCA5 protein truncating mutations are predominantly associated with LCA. However, in two families with the milder EORD, the LCA5 gene analysis revealed a homozygous splice site mutation in one and a stop mutation in combination with a missense mutation in a second family, suggesting that this 
milder phenotype is due to residual function of lebercilin and expanding the currently known phenotypic spectrum to include the milder early onset RP. Some patients have remaining foveal cone structures (intact IS/OS junctions on OCT imaging) and remaining visual acuities, which may bode well for upcoming treatment trials.

Hum Mutat 34:1537-1546, 2013. (C) 2013 Wiley Periodicals, Inc.

KEY WORDS: LCA; RP; retinal dystrophy; blindness; LCA5; lebercilin

\section{Introduction}

Leber congenital amaurosis (LCA) is an infantile onset, severe retinal dystrophy that presents with profound visual impairment, inability to fixate and nystagmus from birth or within the first few weeks of life [von Leber, 1869]. It is the most severe form of a spectrum of disorders arising within the first few years of life, which affect both rod and cone photoreceptors, termed childhood or earlyonset retinal dystrophy (EORD) [Gu et al., 1997]. The term juvenile retinitis pigmentosa (RP) has also been used to describe EORD. Autosomal recessive RP (arRP) falls into this spectrum of disorders with onset typically later in childhood or beyond. LCA accounts for 3\%-5\% of childhood blindness in the developed world and has an incidence of two to three per 100,000 live births [Heckenlively et al., 1988]. Depending on the age at diagnosis, the retinal appearance may be normal or there may be a variety of abnormalities including vascular narrowing, macular atrophy, peripheral white dots at the level of the retinal pigment epithelium (RPE), and retinal pigmentation. The full-field electroretinogram (ERG) is usually nondetectable $(<10 \mu \mathrm{V})$ with conventional ERG testing or severely decreased before the age of 1 year [Foxman et al., 1985; Franceschetti and Dieterle, 1954].

LCA is predominantly inherited as an autosomal recessive trait, although rare dominant forms have been reported [Swaroop et al., 1999]. Nineteen causative genes have thus far been reported: AIPL1 [MIM \#604392] [Sohocki et al., 2000], CABP4 [MIM \#608965] [Aldahmesh et al., 2010], CEP290 [MIM \#610142] [den Hollander et al., 2006], CRB1 [MIM \#604210] [den Hollander et al., 2001], CRX [MIM \#602225] [Freund et al., 1998], GUCY2D [MIM \#600179] [Perrault et al., 1996], IMPDH1 [MIM \#146690] [Bowne et al., 2006], IQCB1 [MIM \#609237] [Estrada-Cuzcano et al., 2011], KCNJ13 [MIM \#603208] [Sergouniotis et al., 2011], LCA5 [MIM \#611408] [den Hollander et al., 2007], LRAT [MIM \#604863] [Thompson et al., 2001], MERTK [MIM \#604705] [Gal et al., 2000], NMNAT1 [MIM \#608700] [Chiang et al., 2012; Falk et al., 2012; Koenekoop et al., 2012; Perrault et al., 2012], RD3 [MIM \#180040] [Friedman et al., 2006], RDH12 [MIM \#608830] [Janecke et al., 2004], RPGRIP1 [MIM \#605446] [Dryja et al., 2001], RPE65 [MIM \#180069] [Gu et al., 1997; Marlhens et al., 1997], SPATA7 [MIM \#609868] [Wang et al., 2009], and TULP1 [MIM \#602280] [Hanein et al., 2004]. Interestingly, mutations in some of these genes, such as CEP290 [Littink et al., 2010], CRB1 [den Hollander et al., 1999], LRAT [Thompson et al., 2001], MERTK [Gal et al., 2000], RPE65 [Morimura et al., 1998], and SPATA7 [Wang et al., 2009], may also be associated with milder forms of retinal dystrophy.

The LCA5 locus (MIM \#604537) was mapped to chromosome 6q11-q16 by traditional linkage studies in a multigenerational kindred of the Old Order River Brethren [Dharmaraj et al., 2000]. Further homozygosity mapping in consanguineous and noncon- sanguineous families led to a refinement of the critical region [den Hollander et al., 2007; Mohamed et al., 2003] and the causative gene, LCA5, was identified [den Hollander et al., 2007]. LCA5 comprises nine exons, seven of which encode Lebercilin, a 697 amino acid protein that contains four coiled-coil domains [den Hollander et al., 2007]. Lebercilin is widely expressed in human tissues including cilia. In situ hybridization of mouse embryos at day 12.5 postcoitum detected almost ubiquitous, low-level staining. At embryonic day 14.5, staining of the eye, inner ear, kidney, and regions of the central and peripheral neural system, gut and the ciliated epithelium of the nasopharynx, trachea, and lungs was more pronounced. In the adult eye, expression was limited to the photoreceptor cell layer. In ciliated cell lines, lebercilin localizes to the ciliary axoneme. In mouse and rat retina, it is found between the outer and inner segments of the photoreceptor layer. In a human embryonic kidney cell line, HEK293, recombinant lebercilin was found to interact with 24 proteins, many of which are associated with centrosomal or ciliary functions, and the intraflagellar transport machinery [Boldt et al., 2011]. Lca5 inactivation in mice led to very severe and rapid retinal degeneration. At 1 month postnatally, there was a significant reduction in photoreceptor cells, and after 4 months, the photoreceptor layer was completely absent [Boldt et al., 2011].

To date, 12 different mutations in LCA5 have been reported. In patients of all families except one, the phenotype has been typical of LCA [Abu-Safieh et al., 2013; Ahmad et al., 2011; den Hollander et al., 2007; Gerber et al., 2007; Jacobson et al., 2009; Li et al., 2011; Ramprasad et al., 2008; Vallespin et al., 2010a; Vallespin et al., 2010b]. Despite its ubiquitous expression and its crucial functions in the cilia [Boldt et al., 2011], the phenotype associated with LCA5 mutations has thus far been confined to the retina. In this paper, we present the results of screening for mutations in LCA5 in a large number of subjects with LCA and EORD $(n=797)$, and arRP $(n=211)$, and describe the associated phenotypes.

\section{Materials and Methods}

\section{Patients}

In total, we sequenced 1,008 patients. We ascertained 797 probands with LCA and EORD, living in Belgium $(n=47)$, Canada and the USA $(n=300)$, Germany $(n=65)$, The Netherlands $(n=54)$, South Africa $(n=18)$, Sweden $(n=20)$, Switzerland $(n=42)$, China $(n=100)$, Pakistan $(n=1)$, and the United Kingdom $(n=150)$. In addition, we included 211 persons with isolated or arRP from The Netherlands. Many LCA and arRP patients were not originally from their current place of residence and were actually from India, Pakistan, Russia, and other countries. Approximately 50\% of the persons with LCA were previously analyzed using various Asper Ophthalmics LCA allele-specific primer extension (APEX) mutation chips. None of the DNAs of persons with arRP were previously analyzed for LCA5 mutations using APEX. LCA, EORD, and RP may be considered a continuum of retinal dystrophies, in which LCA represents the extreme end of severity, as far as the age of onset and functional outcome are concerned. In our current study, LCA was diagnosed in children who were born with severe visual loss, inability to fixate at around 6 weeks of age, nystagmus, and absent or nondetectable ERGs. EORD was diagnosed in children born with reduced, but clinically identifiable, visual function and fixation, with or without nystagmus, and with progressive night blindness and subsequent vision loss. arRP was diagnosed in patients without nystagmus, a variable onset of progressive night blindness and visual loss after the age of 2 years. 


\section{Clinical Investigations}

All patients and family members provided informed consent as part of research projects approved by the local research ethics committees, and the study was conducted in accordance with the principles of the Declaration of Helsinki. Clinical evaluation, including monocular best-corrected visual acuity by projected Snellen charts, slit-lamp biomicroscopy, and fundus examination was performed on all patients who could cooperate with testing. Where possible, patients underwent retinal imaging using a Topcon TRC 501A retinal camera (Topcon Corporation, Tokyo, Japan), optical coherence tomography using a SPECTRALIS ${ }^{\circledR}$ Spectral domain optical coherence tomography (OCT) scanner (Heidelberg Engineering, Heidelberg, Germany), and retinal autofluorescence imaging using a confocal scanning laser ophthalmoscope (Zeiss Prototype; Carl Zeiss Inc., Oberkochen, Germany). Pattern and full-field electroretinogram (PERG and ERG) were performed, where possible, using the recommendations of the International Society for Clinical Electrophysiology of Vision (ISCEV) or a modified Paediatric ERG protocol with skin electrodes as previously described [Flitcroft et al., 2005; Kriss, 1994; Marmor et al., 2009]. Patient \#14 (Berman-Gund Laboratory ID: 048-038) was evaluated by full-field ERG testing using Burian Allen contact lens electrodes, narrow band-passed filtering, and computer averaging that can extend the range of detectability of $30 \mathrm{~Hz}$ cone ERGs to $0.05 \mu \mathrm{V}$ [Berson et al., 1993]. Goldmann perimetry was performed in patient \#16.

Blood samples were collected in EDTA tubes and DNA was extracted using the Puregene blood extraction kit (Invitrogen, Paisley, UK) following manufacturer's instructions, or by a standard salting out procedure [Miller et al., 1988].

\section{Mutation Screening}

Nucleotide numbering reflects cDNA with +1 corresponding to the A of the ATG translation initiation codon (RefSeq NM 181714.3), according to journal guidelines (www.hgvs.org/ mutnomen). The initiation codon is codon 1. Primers used to amplify the coding exons and the intron-exon boundaries of LCA5 were designed using those published previously [den Hollander et al., 2007]. In addition, 194 persons with LCA and 94 persons with arRP were analyzed for the presence of an exon 1 deletion identified in the original LCA5 family [den Hollander et al., 2007]. To this end, primers $5^{\prime}$-TTCCGTGCAATTTAGGGATT- $3^{\prime}$ and $5^{\prime}-$ CGGGGTTTTTGTTTTGTTTG-3' ${ }^{\prime}$ were designed to amplify a deletion breakpoint fragment of $117 \mathrm{bp}$. All standard polymerase chain reactions (PCR) were performed in a total volume of $30 \mu \mathrm{l}$ containing $200 \mu \mathrm{M}$ dNTPs (Bioline, London, UK), $20 \mu \mathrm{M}$ of each primer, $1 \times$ reaction buffer with $1.5 \mathrm{mM} \mathrm{MgCl}_{2}$ (Bioline) with 1 unit of Biotaq (Bioline) and $100 \mathrm{ng}$ of DNA. PCR was carried out on a PTC200 DNA engine thermal cycler (Bio-Rad, Hemel Hempstead, UK). Cycling conditions were as follows: 5 min denaturation at $94^{\circ} \mathrm{C}$ followed by 35 cycles of $94^{\circ} \mathrm{C}$ for $30 \mathrm{sec}$, annealing temperature for $30 \mathrm{sec}$, and extension at $72^{\circ} \mathrm{C}$ for $30 \mathrm{sec}$. A final extension of $72^{\circ} \mathrm{C}$ for $5 \mathrm{~min}$ completed the cycling conditions.

PCR products were visualized on a $2 \%$ agarose gel containing $0.05 \%$ ethidium bromide. The products were cleaned using multiscreen PCR filter plates (Millipore, Watford, UK) prior to sequencing. PCR products were sequenced directly using the ABI Prism Big Dye terminator Kit (V3.1, Applied Biosystems, Foster City, CA) in a $10-\mu 1$ reaction. Samples were purified using the Montage cleanup kit (Millipore) prior to being run on an ABI applied biosystems 3730 DNA sequencer (Applied Biosystems). Electropherograms were analyzed for sequence changes using DNAStar computational software (DNAStar, Inc., Madison, WI). Any sequence changes identified were checked visually.

Mutations were tested in normal controls when possible, and cosegregation was performed in the families, when possible. Mutation frequencies were analyzed in the exome variant server database, 1000 genomes database, and dbSNP database. In silico analyses were then performed to assess pathogenicity, including Blosum62, Polyphen2, and PhyloP.

Patient \#1 was originally identified to harbor a heterozygous mutation in LCA5 by microarray analysis using the Asper LCA Apex chip. His second mutation was identified following LCA5 sequence analysis. Patient \#18 was selected for LCA5 sequence analysis because homozygosity mapping of two affected persons (IV:2 and IV:3) of family W12-2240 (Fig. 1) showed a large, significant homozygous region surrounding LCA5. To identify this region, we had

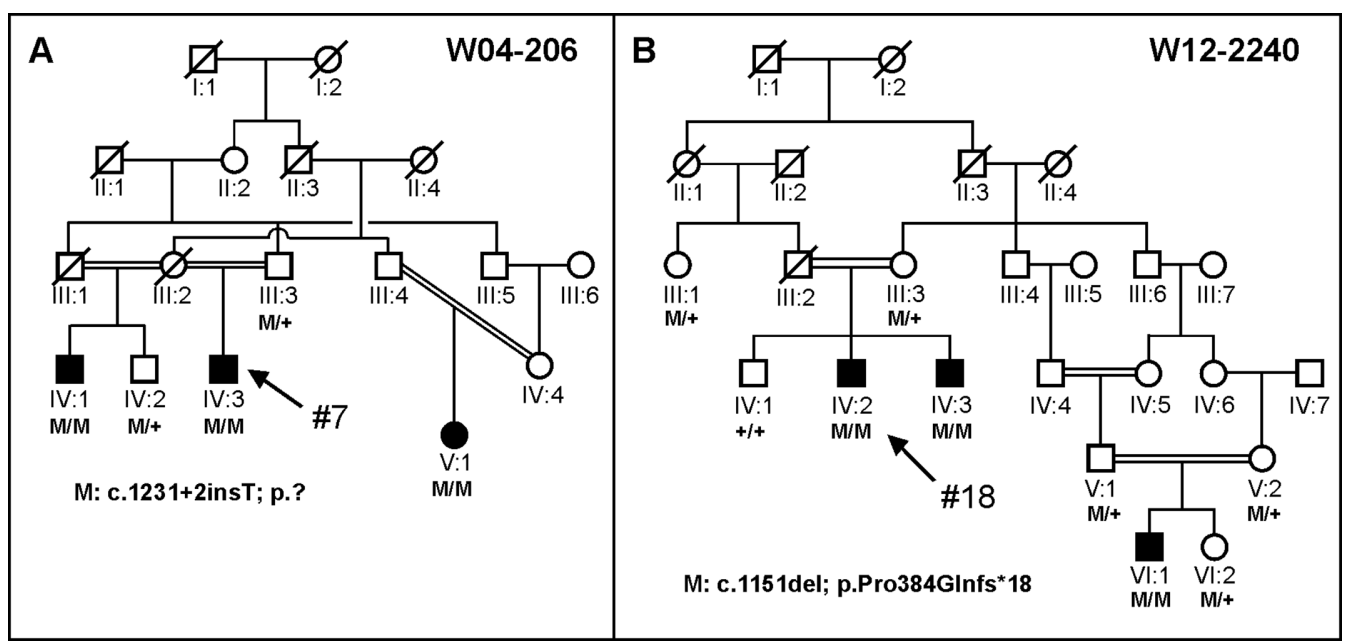

Figure 1. Pedigrees of patients \#7 and \#18. A: An EORD family with proband \#7 is indicated with an arrow. A half-brother and cousin also show the clinical characteristics of arRP and carry the splice variant (M: c.1231+2insT) in the homozygous state. One unaffected brother and the father of the proband carry this variant in the heterozygous state. B: An LCA family with proband \#18. A frequent Pakistani LCA5 variant (c.1151del) is found in the homozygous state in three persons with LCA and in the heterozygous state in five unaffected persons. 
Table 1. Mutations Identified in LCA5

\begin{tabular}{|c|c|c|c|c|c|c|}
\hline \multirow[b]{2}{*}{ Patient } & \multicolumn{2}{|c|}{ Allele 1} & \multicolumn{2}{|c|}{ Allele 2} & \multirow[b]{2}{*}{ Segregation } & \multirow[b]{2}{*}{ Reference (allele 1/allele 2) } \\
\hline & DNA variant & Protein variant & DNA variant & Protein variant & & \\
\hline 1 & c. $835 \mathrm{C}>\mathrm{T}$ & p. $\left(\mathrm{G} \ln 279^{*}\right)$ & c. $1756 \mathrm{~A}>\mathrm{T}$ & p. $\left(\right.$ Lys $\left.586^{*}\right)$ & Yes & den Hollander et al., 2007/novel \\
\hline 2 & c.1151delC & p. $($ Pro384Glnfs*18) & c.1151delC & p. $($ Pro384Glnfs*18) & Yes & den Hollander et al., 2007 \\
\hline 3 & c.633_639del & p.(Glu211Aspfs*13) & c.633_639del & p.(Glu211Aspfs*13) & M-yes; F-NT & Novel \\
\hline 4 & c. $3 \mathrm{G}>\mathrm{A}$ & p.(Met1Ile) & c. $835 \mathrm{C}>\mathrm{T}$ & p. $\left(\mathrm{G} \ln 279^{*}\right)$ & NT & Novel/den Hollander et al., 2007 \\
\hline 5 & c. $1676 \mathrm{C}>\mathrm{A}$ & p. $\left(\right.$ Ser $\left.559^{*}\right)$ & c. $1676 \mathrm{C}>\mathrm{A}$ & p. $\left(\right.$ Ser $\left.559^{*}\right)$ & Yes & Novel \\
\hline 6 & c. $367 \mathrm{C}>\mathrm{T}$ & p. $\left(\mathrm{G} \ln 123^{*}\right)$ & c. $1756 \mathrm{~T}>\mathrm{A}$ & p. $\left(\right.$ Lys $\left.586^{*}\right)$ & NT & Novel/novel \\
\hline 7 & c. $1231+2$ ins $\mathrm{T}$ & p.? & c. $1231+2$ ins $\mathrm{T}$ & p.? & Yes & Novel \\
\hline 8 & c. $142 \mathrm{~A}>\mathrm{T}$ & p. $\left(\operatorname{Arg} 48^{*}\right)$ & c. $142 \mathrm{~A}>\mathrm{T}$ & p. $\left(\operatorname{Arg} 48^{*}\right)$ & NT & Novel \\
\hline 9 & c. $142 \mathrm{~A}>\mathrm{T}$ & p. $\left(\operatorname{Arg} 48^{*}\right)$ & c. $142 \mathrm{~A}>\mathrm{T}$ & p. $\left(\operatorname{Arg} 48^{*}\right)$ & NT & Novel \\
\hline 10 & c.1543_1544delinsA & p.(Ser515Lysfs*78) & c.1543_1544delinsA & p.(Ser515Lysfs*78) & NT & Novel \\
\hline 11 & c. $795 \mathrm{~T}>\mathrm{G}$ & p. $\left(\operatorname{Tyr} 265^{*}\right)$ & c.1759_1760insAG & p.(Leu587Glnfs*7) & NT & Novel/novel \\
\hline 12 & c. $835 \mathrm{C}>\mathrm{T}$ & p. $\left(\mathrm{G} \ln 279^{*}\right)$ & c. $835 \mathrm{C}>\mathrm{T}$ & p. $\left(\mathrm{G} \ln 279^{*}\right)$ & NT & den Hollander et al., 2007 \\
\hline 13 & c. $42 \_45 \mathrm{del}$ & p.(Lys15Glnfs*95) & c. $1207 \mathrm{C}>\mathrm{T}$ & p. $\left(\mathrm{G} \ln 403^{*}\right)$ & NT & Novel/novel \\
\hline 14 & c.1730dup & p.(Leu577Phefs*12) & + & + & NT & Novel \\
\hline 15 & c. $2011 \mathrm{C}>\mathrm{T}$ & p. $\left(\operatorname{Arg} 671^{*}\right)$ & + & + & NT & Novel \\
\hline 16 & c. $69 \mathrm{C}>\mathrm{G}$ & p. $\left(\right.$ Tyr $\left.23^{*}\right)$ & c. $491 \mathrm{~A}>\mathrm{G}$ & p.(His164Arg) & NT & Novel/novel \\
\hline 17 & c.439_449dup & p. $($ Glu151*) & c.439_449dup & p. $\left(\right.$ Glu $\left.151^{*}\right)$ & Yes & Novel \\
\hline 18 & c.1151delC & p. $($ Pro384Glnfs*18) & c.1151delC & p. $($ Pro384Glnfs*18) & Yes & den Hollander et al., 2007 \\
\hline
\end{tabular}

Mutation nomenclature is based on NM_181714.3.

F, father; M, mother; NT, not tested; +, not identified.

employed the CytoScan ${ }^{\circledR}$ High-Density array containing probes to detect 750,000 single-nucleotide polymorphisms (Affymetrix, Santa Clara, CA).

\section{Results}

\section{Mutational Analysis}

A total of 797 patients with LCA and EORD and 211 with arRP were screened for sequence variants in protein-coding exons (i.e., exons 3 through 9) of $L C A 5$. Sixteen probands were identified with two disease-causing variants. In all, 19 different $L C A 5$ variants were identified (Table 1); 17 of these variants are novel. Homozygous variants were found in 10 probands, compound heterozygous variants in six probands, and heterozygous protein truncating variants in two probands (Table 1).

All but two mutations were predicted to lead to premature termination of the lebercilin protein. One of the missense mutations that we identified was in patient \#4 involving the first amino acid of the lebercilin protein (p.Met1Ile). The other missense change is a novel change in patient \#16 involving a histidine to arginine change at position 164 (p.His164Arg). The underlying c.491A $>\mathrm{G}$ variant was not found in 8,600 alleles of "control" individuals in the Exome Variant Server database and once in the 1000 genomes database (rs183669161). Unfortunately, we do not have parental DNA to test for segregation. The PhyloP score of c.491A is 3.92, Polyphen2 predicts damage, and the histidine at position 164 is fully conserved between human, rhesus, mouse, dog, opossum, chicken, Western clawed frog, and zebrafish. In an affected half-brother (IV:1) and an affected cousin (V:1) of patient \#7 (Fig. 1A), the same homozygous splice site variant (c.1231+2insT) was found, and two unaffected persons carried this variant in the heterozygous state. This variant is predicted to completely inactivate this splice site based on SpliceSiteFinder, MaxEntSplice, and NNSplice analysis. Homozygosity mapping using DNA from two persons with LCA from family W12-2240 (Fig. 1B: patients IV:2 and IV:3) yielded 10 shared homozygous regions larger than $5 \mathrm{Mb}$. The third largest homozygous region on chromosome $6(18.9 \mathrm{Mb})$ encompassed LCA5. In all three affected individuals (Fig. 1B), the c.1151delC (p.Pro384Glnfs*18) variant was identified in the homozygous state in LCA5. This variant was present in the heterozygous state in five unaffected members (Fig. 1B).

The ethnic origins of the 18 probands with LCA5 mutations were as follows: European Caucasian: 7 (British, Slovakian-Romani, and Spanish); Asian: 7 (Pakistan, Afghanistan, Indian-Mauritian, Iraqi, Taiwanese, and Chinese); North American: 3; Mexican: 1.

\section{Clinical Phenotype}

Of the 18 probands with LCA5 variants identified in this study, all but two patients had a clinical diagnosis of LCA, with onset of severe visual loss at birth or within the first few months of life, and nystagmus (Table 2). However, patients \#7 and \#16 received a diagnosis of EORD. Patient \#7 was born with congenital nystagmus, and developed night blindness at the age of 3 , visual field loss at the age of 6 , and visual loss at the age of 8 . In patient \#16, the visual loss started after the age of 40 years. In addition, patient \#16 did not have nystagmus. All of our LCA and EORD patients had nyctalopia and severe visual field constriction. General health was good in the majority of patients, except in patient \#2 who had global developmental delay and patient \#3 who had behavioral problems. Fourteen of the 16 LCA patients were legally blind with visual acuity ranging from $1.0 \operatorname{logMAR}$ to light perception. One patient, \#11, with LCA and nystagmus, had visual acuities of 0.48 right (20/60) and 0.54 left (20/70) at the age of 6 years (Table 2). Patient \#16, with EORD, had a visual acuity of $0.30(20 / 40)$ at the age of 55 years. Most patients had a hypermetropic refraction (Table 2). The high myopic correction in patient \#6 may be attributed to keratoconus. A fullfield ERG was performed in eight patients and was nondetectable $(<10 \mu \mathrm{V})$ with conventional ERG testing in five patients. A small cone ERG response could be quantified in patient \#14 and trace cone ERG responses (unquantified) could be detected in patient \#16 (Table 2). Anterior segments were normal in all but one of the oldest patients (patient \#6), who had bilateral keratoconus at the age of 37 years. This patient also developed bilateral nuclear sclerotic and posterior subcapsular lenticular opacities in adulthood.

Fundus examination most commonly revealed widespread retinal pigment epithelial atrophy and granularity, with peculiar, small, white dots at the level of the RPE in the retinal periphery, which 


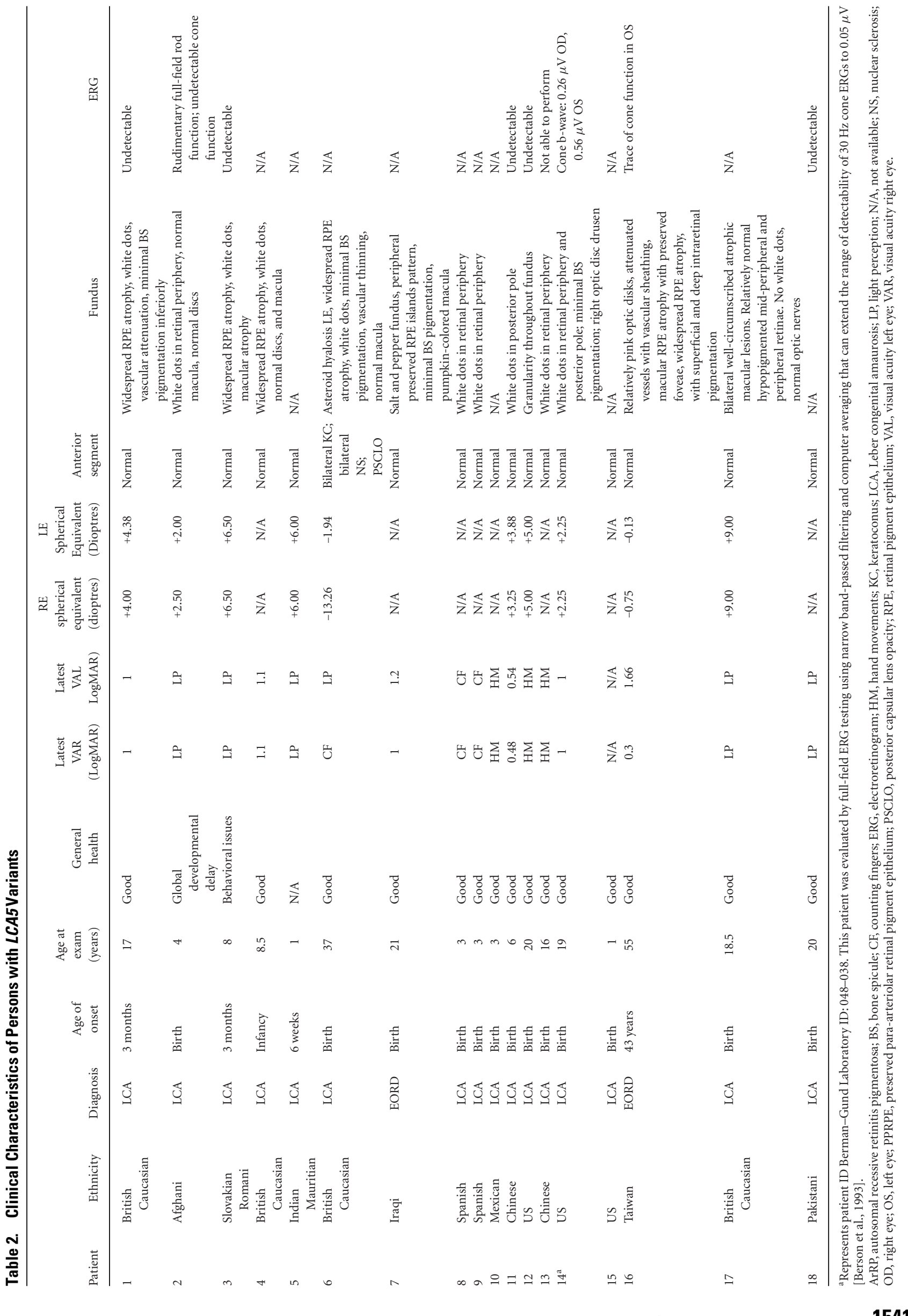



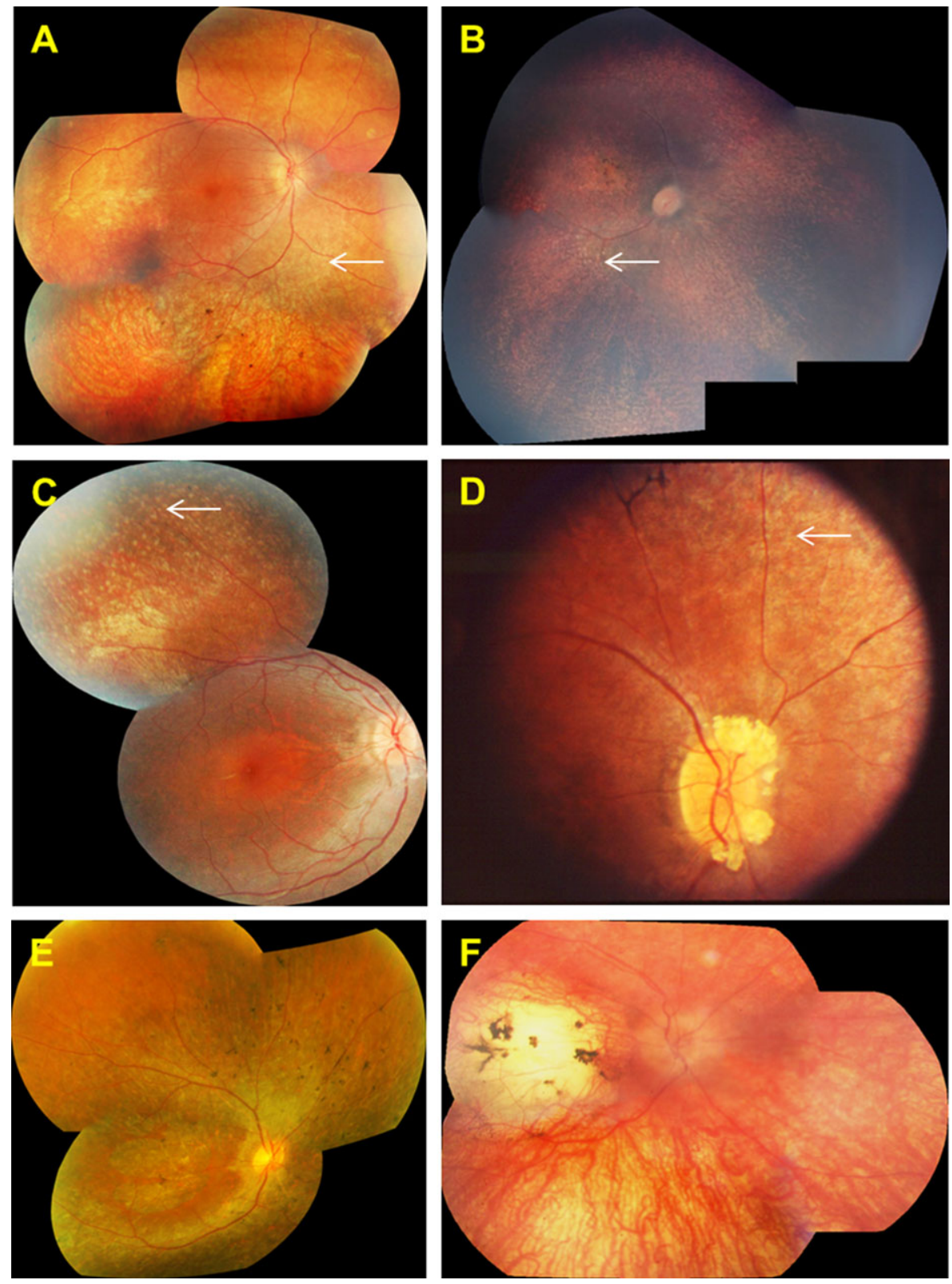

Figure 2. Retinal phenotype of patients with $L C A 5$ mutations. Right eye shown for each patient. A: Patient \#1, age 17 years, with widespread RPE atrophy, mid-peripheral retinal white dots (white arrow), and peripheral intraretinal pigmentation B: Patient \#3, age 5 years, with mid-peripheral retinal white dots (white arrow) and macular atrophy. C: Patient \#4, age 8.5 years, with widespread RPE atrophy and mid-peripheral retinal white dots (white arrow). D: Patient \#14, age 19 years, with retinal white dots (white arrow), peripheral intraretinal pigmentation, and optic disk drusen. E: Patient \#16, age 55 years, with widespread RPE atrophy, foveal preservation, and peripheral intraretinal pigmentation. F: Patient \#17, age 18.5 years, with marked macular atrophy and hypopigmented but relatively normal peripheral retina. Intraretinal pigment was seen in the oldest patients (panels $A, D$, and $E$ ).

were seen in 10 of 14 patients $(71 \%)$ in whom funduscopic data were available (Fig. 2). Figure $2 \mathrm{~B}$ and $\mathrm{C}$ shows the white dots clearly, and document the round, evenly spaced, similar-sized lesions. Intraretinal pigment migration, when present, was minimal, situated in the far retinal periphery, and seen in the oldest patients, patients $\# 1, \# 6, \# 7, \# 14$, and \#16 (Fig. 2A, D, and E). Macular atrophy was noted in only three patients, \#3, \#16, and \#17 (Fig. 2B, E, and F); the macula was otherwise normal on funduscopy in the remaining patients. In one patient, the macular lesion was particularly severe, with features of a "macular coloboma" (Fig. 2F). Optic discs were normal in appearance (pink disc color) in all but one patient (patient
\#14), who had optic disc drusen (Fig. 2D). In one family with arRP (patient \#7), we identified a pumpkin-colored maculopathy and intraretinal pigmentation in the periphery with faintly preserved islands of RPE (Fig. 3).

OCT data, available for patients \#1, \#11, and \#16, showed preservation of the central foveal inner segment/outer segment (IS/OS) junctions and of the outer segments in the two younger patients (\#1 and \#11) (Fig. 4A and B). In the older patient (\#16), OCT imaging demonstrated macular atrophy, disruption of retinal lamination, and the presence of a hyporeflective well-circumscribed area in the outer nuclear layer, with a hyperreflective border. This may 


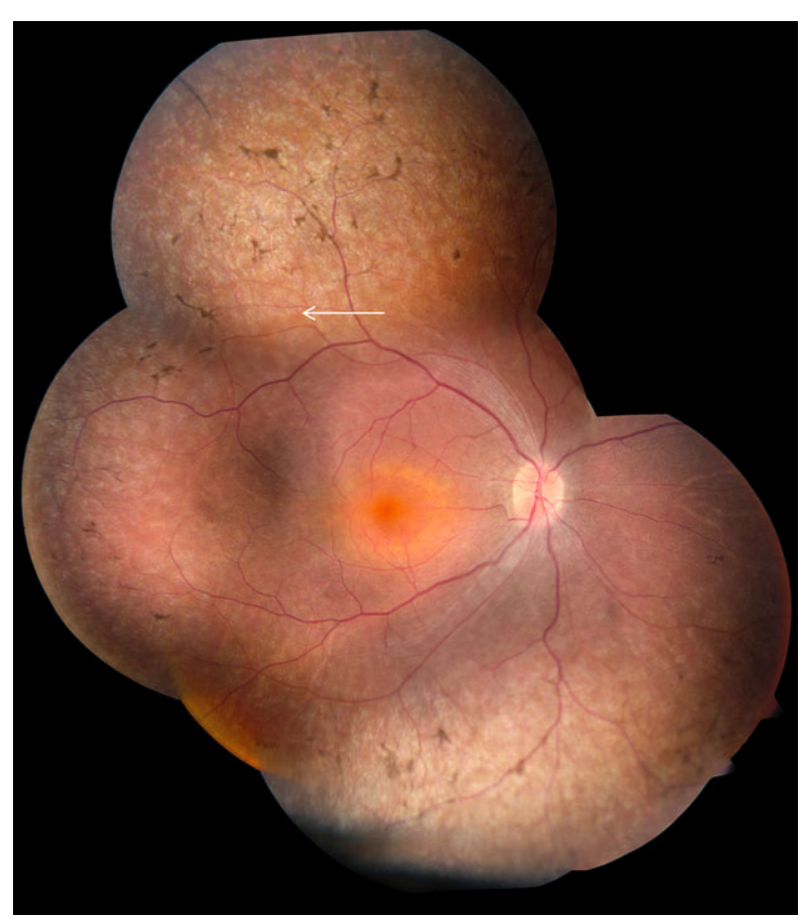

Figure 3. Retinal phenotype of arRP patient \#7, right eye, age 21 years. There is widespread RPE atrophy, peripheral preserved islands of RPE (white arrow), peripheral intraretinal pigmentation, and a "pumpkin"colored macula.

represent an area of outer retinal tubulation (also known as rosettes) (Fig. 4C). OCT image acquisition was otherwise not possible due to nystagmus. Fundus autofluorescence (FAF) imaging data were available in two patients (Fig. 5). FAF in patient \#1 revealed an overall hypofluorescence in the macula, with a central hyperfluorescent signal in the fovea (Fig. 5A). FAF images of patient \#16 showed severe hypofluorescence in the macula corresponding to significant RPE atrophy (Fig. 5B). Goldmann perimetry in patient \#16 revealed a small central island, with a relatively large preserved nasal field and a small temporal field.

\section{Discussion}

LCA5 mutations are a rare cause of LCA. Previously, only 12 different mutations had been identified and published in 16 families with LCA. In the current study, which is the largest study to date, we have screened the coding region of LCA5 in 1,008 patients, 797 with LCA and EORD, and 211 with arRP, ascertained from eight different countries. We identified mutations in 18 new LCA families. We discovered 19 different LCA5 mutations, 17 of which are novel. Mutations in LCA5 thus account for $\sim 2 \%$ of patients with LCA, EORD, and arRP in our cohort. All 29 different LCA5 variants have now been included in the new Leiden Open Variation Database (LOVD; http://www.LOVD.nl/LCA5). In two patients (\#14 and \#15), we identified heterozygous variants. The second allele may have been missed because the variant may be located outside the protein-coding exons, or because these variants are causative in a more complex inheritance model (such as digenic), or that these are coincidental findings of heterozygous variants that play no role in the LCA phenotype.
The majority of LCA5 mutations found both in this study and in those published previously are likely null mutations due to premature termination of the protein. Only one missense mutation in this gene (p.Ser202Pro) has been previously reported in LCA, but without accompanying clinical information [Vallespin et al., 2010b]. We also identified a missense variant, p.Met1Ile (the start codon), in patient \#4, who carried a diagnosis of LCA, in combination with the most common LCA5 stop mutation p.Gln279*. If the next methionine in the open reading frame were to be used instead (at position 336), as the alternative start codon, then two coiled-coiled domains in the lebercilin protein would be lacking, very likely rendering the truncated lebercilin inactive. Therefore, this missense mutation may also be a null allele. We also identified another missense mutation, p.His164Arg. This mutation was found in combination with a novel nonsense mutation, p.Tyr23*, in LCA patient \#16, but segregation data are lacking. Thus, we cannot exclude that these variants are in cis configuration and that they are not causative for the phenotype in patient \#16.

Previously, and in the majority of patients in this study, the phenotype reported in association with LCA5 mutations is consistent with LCA with severely reduced vision at, or near birth, nystagmus, and a nondetectable $(<10 \mu \mathrm{V})$ ERG [Ahmad et al., 2011; den Hollander et al., 2007; Dharmaraj et al., 2000; Gerber et al., 2007; Jacobson et al., 2009; Mohamed et al., 2003; Ramprasad et al., 2008]. High hypermetropia is common. The visual acuity is reported to range between 0.20 to light perception and there is extensive peripheral field loss. In our study, the majority of patients had a clinical diagnosis of LCA, with fundus examination revealing widespread atrophy of the retina and RPE but with little intraretinal pigment migration. On the clinical examination, we commonly identified scattered white dots at the level of the RPE (Fig. 2A-D-white arrows). White dots can be found in other genetic types of LCA, such as RPE65, LRAT, and CEP290. In RPE65-type LCA, Weleber et al. (2011) suggested that the white dots might represent accumulations of trans-retinyl esters. The biochemical make-up of the white dots associated with LCA5 mutations is as yet unknown. Our LCA5-associated retinal phenotypes are consistent with the retinal changes observed in other patients with LCA5 mutations (Figs. 2 and 3) [Ahmad et al., 2011; Dharmaraj et al., 2000; Gerber et al., 2007; Mohamed et al., 2003; Ramprasad et al., 2008].

However, in this study, for the first time, we identified an Iraqi family with two affected sibs who carried a diagnosis of EORD, because they maintained some useful vision until the age of 10 years. In addition, these siblings had preservation of the peripheral islands of RPE (Fig. 3), a phenotype similar to preserved paraarteriolar retinal pigment epithelium documented by Heckenlively (1982) and later found to be associated with LCA and arRP patients with CRB1 mutations [den Hollander et al., 1999; Heckenlively, 1982]. We have thus expanded the phenotypic spectrum associated with LCA5 mutations, which now includes both EORD and a new retinal phenotype consisting of preserved islands of peripheral RPE (Fig. 3).

There is little published data on retinal imaging such as OCT and FAF imaging in patients with $L C A 5$ mutations. In this regard, we here contribute three new patients with LCA5 mutations and new OCT and FAF data. However, it is a limitation of the present study that we were unable to obtain good quality autofluorescence imaging and OCT on the entire cohort. This was, in part, due to the inherent difficulties of such imaging in patients with nystagmus, their ages and their remote living conditions. Jacobson et al. (2009) reported a low autofluorescent signal corresponding to macular atrophy in two siblings with a homozygous LCA5 mutation, in whom mutations in CRB1 had previously been excluded. We demonstrated similar 

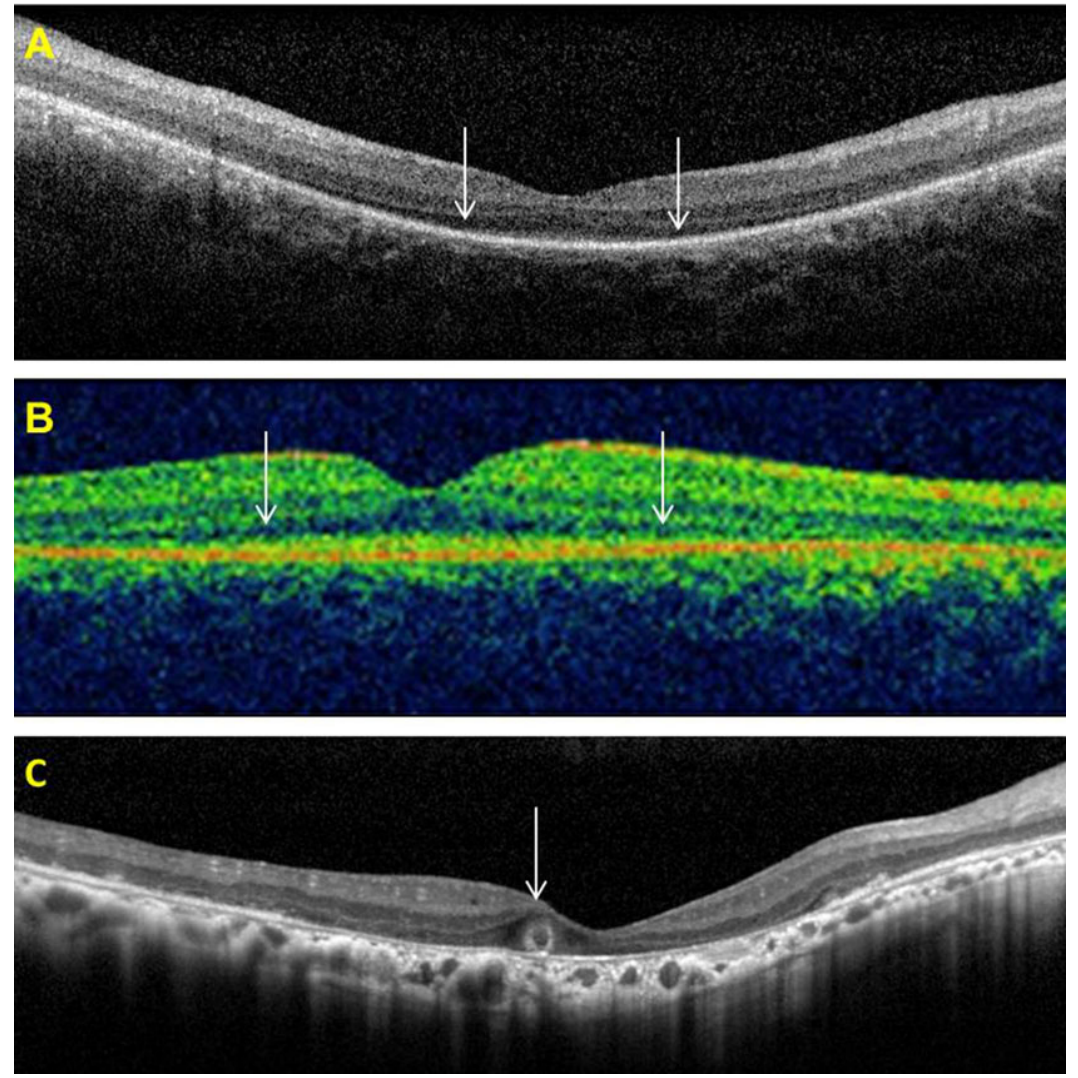

Figure 4. Optical coherence tomography imaging of patients with $L C A 5$ variants. A: Patient \#1 (age 17 years). B: Patient \#11 (age 6 years)—white arrows demarcate the extent of preservation of the central foveal IS/OS junctions and of the outer segments. C: Patient \#16 (age 55 years) demonstrating macular atrophy, disruption of retinal lamination, and a presumed area of outer retinal tubulation (white arrow).

macular hypoautofluorescence in one subject who in addition had a small area of hyperautofluorescence at the fovea, suggesting continued metabolic activity of the foveal cones and RPE. In our study, OCT imaging was possible in three subjects, and the findings revealed surprising preservation of the central foveal IS/OS junctions and of the outer segment structures (in the younger patients, of the age of 17 years and 6 years, respectively). In the older patient, of the age of 55 years, OCT imaging demonstrated macular atrophy, loss of the retinal lamination, and probable outer retinal tubulation (also known as rosettes), a late-stage process in the degeneration
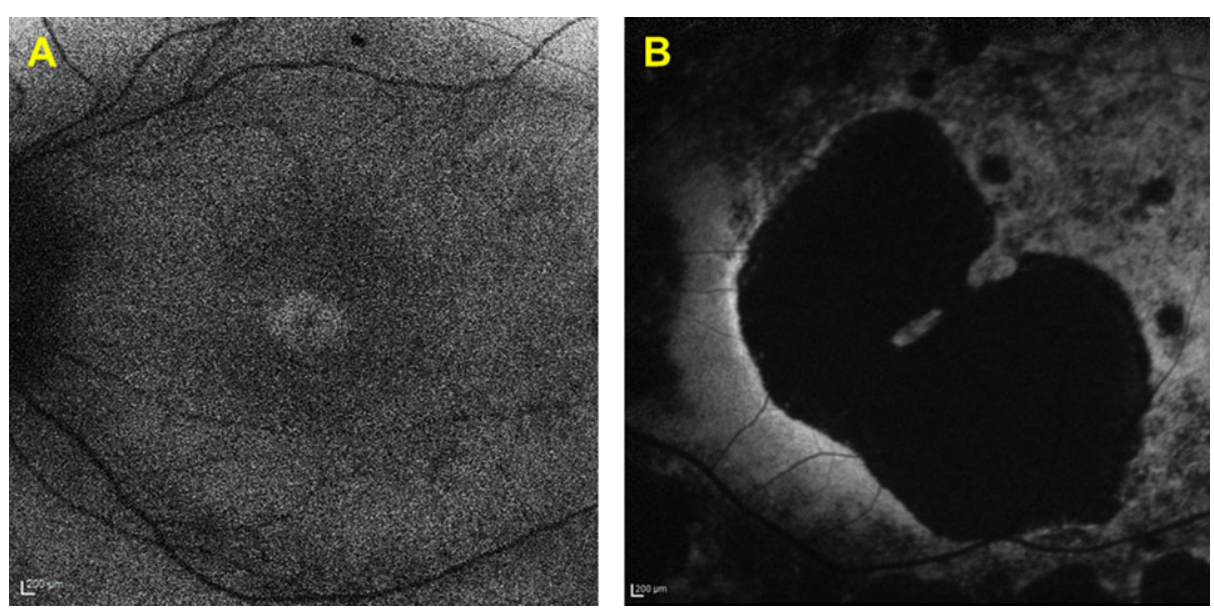

Figure 5. Fundus autofluorescence imaging of patients with $L C A 5$ variants. A: Patient \#1 (age 17 years), demonstrating overall macular hypofluorescence, with a hyperfluorescent foveal signal, suggesting metabolic activity of the foveal cones and RPE complex. B: Patient \#16 (age 55 years), showing a large area of macular hypofluorescence corresponding to RPE atrophy. 
of photoreceptors that has been described recently in a number of retinal conditions (Fig. 4C) [Sergouniotis et al., 2012; Zweifel et al., 2009]. Previously published OCT findings have demonstrated loss of the photoreceptor and outer nuclear layers, and abnormal retinal lamination eccentric to the fovea, but with evidence of a retained photoreceptor layer at the fovea [Jacobson et al., 2009]. This suggests that in the early stages of the disease, there may be viable photoreceptors (cones) in the foveal region, which may be amenable to therapeutic intervention. It is of interest that patient $\# 14$ at the of age of 19 , with an advanced stage of disease, still had a detectable $30 \mathrm{~Hz}$ cone ERG in each eye (Table 2) with narrow band-passed filtering and computer averaging. In future clinical trials, this technique of ERG recording, which is not performed in the present ISCEV protocol, could be considered as a method of objectively monitoring efficacy or lack of efficacy as undoubtedly most patients with remaining vision will have a detectable cone ERG with this technique.

Lebercilin is expressed in a number of tissues; however, there have been no reports of extraocular abnormalities in LCA5 mutations. One patient with LCA5 retinopathy died from asphyxia and the authors propose a possible link between the cause of death and the presence of lebercilin in the bronchial ciliated epithelial cells [Ramprasad et al., 2008]. In the present study, one patient had global developmental delay, whereas another experienced behavioral problems. Whether this is related to abnormal extraocular expression of lebercilin remains to be determined.

LCA5-associated LCA is a rare form of severe childhood onset retinal dystrophy. Most LCA5 mutations are null and are associated with a severe phenotype. Missense mutations are rare and may be associated with a milder EORD, suggesting that such mutations may result in some residual protein function. LCA5-type LCA may be amenable to treatment, but this is more likely to be successful in young patients.

\section{URLs Used in This Study}

Exome Variant Server database:

http://evs.gs.washington.edu/EVS/

1000 genomes database:

http://www.1000genomes.org/

PhyloP:

http://www.ncbi.nlm.nih.gov/pmc/articles/PMC2808870/

Polyphen2:

http://genetics.bwh.harvard.edu/pph2/

SpliceSiteFinder:

www.genet.sickkids.on.ca/ ali/splicesitefinder.html

MaxEntSplice:

http://genes.mit.edu/burgelab/maxent/Xmaxentscan_scoreseq.

html

NNSplice:

http://www.fruitfly.org/seq_tools/splice.html

\section{Acknowledgments}

We sincerely thank the patients' families for taking part in this study. We are grateful for those who contributed to the assembly of large panels of probands, particularly in London, Bev Scott, Genevieve Wright, and Sophie Devery. The LCA5 Study Group consists of Sten Andreasson (Skane University Hospital Lund), Elfride de Baere (Ghent University), Jean Bennett (Scheie Eye Institute Philadelphia), Gerry Chader (Ophthalmology, Doheny Eye Institute, Keck School of Medicine, University of Southern California, Los Angeles, California), Wolfgang Berger (University of Zurich), Irina Golovleva (Umeå University), Jacquie Greenberg (Institute of Infectious
Disease and Molecular Medicine, University of Cape Town), Anneke I. den Hollander (Radboud University Nijmegen Medical Centre), Caroline C.W. Klaver (Erasmus Medical Center, Rotterdam), B. Jeroen Klevering (Radboud University Nijmegen Medical Centre), Birgit Lorenz (Justus Liebig University Gie $\beta$ en), Markus N. Preising (Justus Liebig University Gie $\beta$ en), Raj Ramesar (Institute of Infectious Disease and Molecular Medicine, University of Cape Town), Lisa Roberts (Institute of Infectious Disease and Molecular Medicine, University of Cape Town), Ronald Roepman (Radboud University Nijmegen Medical Centre), Klaus Rohrschneider (Universitäts-Augenklinik Heidelberg), and Bernd Wissinger (University of Tübingen).

\section{References}

Abu-Safieh L, Alrashed M, Anazi S, Alkuraya H, Khan AO, Al-Owain M, Al-Zahrani J, Al-Abdi L, Hashem M, Al-Tarimi S, Sebai MA, Shamia A, et al. 2013. Autozygomeguided exome sequencing in retinal dystrophy patients reveals pathogenetic mutations and novel candidate disease genes. Genome Res 23:236-247.

Ahmad A, Daud S, Kakar N, Nurnberg G, Nurnberg P, Babar ME, Thoenes M, Kubisch C, Ahmad J, Bolz HJ. 2011. Identification of a novel LCA5 mutation in a Pakistani family with Leber congenital amaurosis and cataracts. Mol Vis 17:1940-1945.

Aldahmesh MA, Al-Owain M, Alqahtani F, Hazzaa S, Alkuraya FS. 2010. A null mutation in CABP4 causes Leber's congenital amaurosis-like phenotype. Mol Vis 16:207-212.

Berson EL, Rosner B, Sandberg MA, Hayes KC, Nicholson BW, Weigel-DiFranco C, Willett W. 1993. A randomized trial of vitamin A and vitamin E supplementation for retinitis pigmentosa. Arch Ophthalmol 111:761-772.

Boldt K, Mans DA, Won J, van Reeuwijk J, Vogt A, Kinkl N, Letteboer SJ, Hicks WL, Hurd RE, Naggert JK, Texier Y, den Hollander AI, et al. 2011. Disruption of intraflagellar protein transport in photoreceptor cilia causes Leber congenital amaurosis in humans and mice. J Clin Invest 121:2169-2180.

Bowne SJ, Sullivan LS, Mortimer SE, Hedstrom L, Zhu J, Spellicy CJ, Gire AI, Hughbanks-Wheaton D, Birch DG, Lewis RA, Heckenlively JR, Daiger SP. 2006. Spectrum and frequency of mutations in IMPDH1 associated with autosomal dominant retinitis pigmentosa and leber congenital amaurosis. Invest Ophthalmol Vis Sci 47:34-42.

Chiang PW, Wang J, Chen Y, Fu Q, Zhong J, Yi X, Wu R, Gan H, Shi Y, Barnett C, Wheaton D, Day M, et al. 2012. Exome sequencing identifies NMNAT1 mutations as a cause of Leber congenital amaurosis. Nat Genet 44:972-974.

den Hollander AI, Heckenlively JR, van den Born LI, de Kok YJ, van der Velde-Visser SD, Kellner U, Jurklies B, van Schooneveld MJ, Blankenagel A, Rohrschneider K, Wissinger B, Cruysberg JR, et al. 2001. Leber congenital amaurosis and retinitis pigmentosa with coats-like exudative vasculopathy are associated with mutations in the crumbs homologue 1 (CRB1) gene. Am J Hum Genet 69:198-203.

den Hollander AI, Koenekoop RK, Mohamed MD, Arts HH, Boldt K, Towns KV, Sedmak T, Beer M, Nagel-Wolfrum K, McKibbin M, Dharmaraj S, Lopez I, et al. 2007. Mutations in LCA5, encoding the ciliary protein lebercilin, cause Leber congenital amaurosis. Nat Genet 39:889-895.

den Hollander AI, Koenekoop RK, Yzer S, Lopez I, Arends ML, Voesenek KE, Zonneveld MN, Strom TM, Meitinger T, Brunner HG, Hoyng CB, van den Born LI, et al. 2006. Mutations in the CEP290 (NPHP6) gene are a frequent cause of Leber congenital amaurosis. Am J Hum Genet 79:556-561.

den Hollander AI, ten Brink JB, de Kok YJ, van Soest S, van den Born LI, van Driel MA, van de Pol DJ, Payne AM, Bhattacharya SS, Kellner U, Hoyng CB, Westerveld A, et al. 1999. Mutations in a human homologue of Drosophila crumbs cause retinitis pigmentosa (RP12). Nat Genet 23:217-221.

Dharmaraj S, Li Y, Robitaille JM, Silva E, Zhu D, Mitchell TN, Maltby LP, BaffoeBonnie AB, Maumenee IH. 2000. A novel locus for Leber congenital amaurosis maps to chromosome 6q. Am J Hum Genet 66:319-326.

Dryja TP, Adams SM, Grimsby JL, McGee TL, Hong DH, Li T, Andreasson S, Berson EL. 2001. Null RPGRIP1 alleles in patients with Leber congenital amaurosis. Am J Hum Genet 68:1295-1298.

Estrada-Cuzcano A, Koenekoop RK, Coppieters F, Kohl S, Lopez I, Collin RW, De Baere EB, Roeleveld D, Marek J, Bernd A, Rohrschneider K, van den Born LI, et al. 2011. IQCB1 mutations in patients with leber congenital amaurosis. Invest Ophthalmol Vis Sci 52:834-839.

Falk MJ, Zhang Q, Nakamaru-Ogiso E, Kannabiran C, Fonseca-Kelly Z, Chakarova C, Audo I, Mackay DS, Zeitz C, Borman AD, Staniszewska M, Shukla R, et al. 2012. NMNAT1 mutations cause Leber congenital amaurosis. Nat Genet 44:1040-1045.

Flitcroft DI, Adams GG, Robson AG, Holder GE. 2005. Retinal dysfunction and refractive errors: an electrophysiological study of children. Br J Ophthalmol 89:484-488.

Foxman SG, Heckenlively JR, Bateman JB, Wirtschafter JD. 1985. Classification of congenital and early onset retinitis pigmentosa. Arch Ophthalmol 103: $1502-1506$ 
Franceschetti A, Dieterle P. 1954. [Diagnostic and prognostic importance of the electroretinogram in tapetoretinal degeneration with reduction of the visual field and hemeralopia]. Confin Neurol 14(2-3):184-186.

Freund CL, Wang QL, Chen S, Muskat BL, Wiles CD, Sheffield VC, Jacobson SG, McInnes RR, Zack DJ, Stone EM. 1998. De novo mutations in the CRX homeobox gene associated with Leber congenital amaurosis. Nat Genet 18:311-312.

Friedman JS, Chang B, Kannabiran C, Chakarova C, Singh HP, Jalali S, Hawes NL, Branham K, Othman M, Filippova E, Thompson DA, Webster AR, et al. 2006. Premature truncation of a novel protein, RD3, exhibiting subnuclear localization is associated with retinal degeneration. Am J Hum Genet 79:1059-1070.

Gal A, Li Y, Thompson DA, Weir J, Orth U, Jacobson SG, Apfelstedt-Sylla E, Vollrath D. 2000. Mutations in MERTK, the human orthologue of the RCS rat retinal dystrophy gene, cause retinitis pigmentosa. Nat Genet 26:270-271.

Gerber S, Hanein S, Perrault I, Delphin N, Aboussair N, Leowski C, Dufier JL, Roche O, Munnich A, Kaplan J, Rozet JM. 2007. Mutations in LCA5 are an uncommon cause of Leber congenital amaurosis (LCA) type II. Hum Mutat 28:1245.

Gu SM, Thompson DA, Srikumari CR, Lorenz B, Finckh U, Nicoletti A, Murthy KR, Rathmann M, Kumaramanickavel G, Denton MJ, Gal A. 1997. Mutations in RPE65 cause autosomal recessive childhood-onset severe retinal dystrophy. Nat Genet 17:194-197.

Hanein S, Perrault I, Gerber S, Tanguy G, Barbet F, Ducroq D, Calvas P, Dollfus H, Hamel C, Lopponen T, Munier F, Santos L, et al. 2004. Leber congenital amaurosis: comprehensive survey of the genetic heterogeneity, refinement of the clinical definition, and genotype-phenotype correlations as a strategy for molecular diagnosis. Hum Mutat 23:306-317.

Heckenlively JR. 1982. Preserved para-arteriole retinal pigment epithelium (PPRPE) in retinitis pigmentosa. Br J Ophthalmol 66:26-30.

Heckenlively JR, Foxman SG, Parelhoff ES. 1988. Retinal dystrophy and macular coloboma. Doc Ophthalmol 68(3-4):257-271.

Jacobson SG, Aleman TS, Cideciyan AV, Sumaroka A, Schwartz SB, Windsor EA, Swider M, Herrera W, Stone EM. 2009. Leber congenital amaurosis caused by Lebercilin (LCA5) mutation: retained photoreceptors adjacent to retinal disorganization. Mol Vis 15:1098-1106.

Janecke AR, Thompson DA, Utermann G, Becker C, Hubner CA, Schmid E, McHenry CL, Nair AR, Ruschendorf F, Heckenlively J, Wissinger B, Nurnberg P, et al. 2004 Mutations in RDH12 encoding a photoreceptor cell retinol dehydrogenase cause childhood-onset severe retinal dystrophy. Nat Genet 36:850-854.

Koenekoop RK, Wang H, Majewski J, Wang X, Lopez I, Ren H, Chen Y, Li Y, Fishman GA, Genead M, Schwartzentruber J, Solanki N, et al. 2012. Mutations in NMNAT1 cause Leber congenital amaurosis and identify a new disease pathway for retinal degeneration. Nat Genet 44:1035-1039.

Kriss A. 1994. Skin ERGs: their effectiveness in paediatric visual assessment, confounding factors, and comparison with ERGs recorded using various types of corneal electrode. Int J Psychophysiol 16(2-3):137-146.

Li L, Xiao X, Li S, Jia X, Wang P, Guo X, Jiao X, Zhang Q, Hejtmancik JF. 2011. Detection of variants in 15 genes in 87 unrelated Chinese patients with Leber congenital amaurosis. PloS One 6:e19458.

Littink KW, Pott JW, Collin RW, Kroes HY, Verheij JB, Blokland EA, de Castro Miro M, Hoyng CB, Klaver CC, Koenekoop RK, Rohrschneider K, Cremers FP, et al. 2010. A novel nonsense mutation in CEP290 induces exon skipping and leads to a relatively mild retinal phenotype. Invest Ophthalmol Vis Sci 51:3646-3652.

Marlhens F, Bareil C, Griffoin JM, Zrenner E, Amalric P, Eliaou C, Liu SY, Harris E, Redmond TM, Arnaud B, Claustres M, Hamel CP. 1997. Mutations in RPE65 cause Leber's congenital amaurosis. Nat Genet 17:139-141.

Marmor MF, Fulton AB, Holder GE, Miyake Y, Brigell M, Bach M. 2009. ISCEV Standard for full-field clinical electroretinography (2008 update). Doc Ophthalmol 118:69-77.

Miller SA, Dykes DD, Polesky HF. 1988. A simple salting out procedure for extracting DNA from human nucleated cells. Nucleic Acids Res 16:1215.
Mohamed MD, Topping NC, Jafri H, Raashed Y, McKibbin MA, Inglehearn CF. 2003. Progression of phenotype in Leber's congenital amaurosis with a mutation at the LCA5 locus. Br J Ophthalmol 87:473-475.

Morimura H, Fishman GA, Grover SA, Fulton AB, Berson EL, Dryja TP. 1998. Mutations in the RPE65 gene in patients with autosomal recessive retinitis pigmentosa or leber congenital amaurosis. Proc Natl Acad Sci USA 95:3088-3093.

Perrault I, Hanein S, Zanlonghi X, Serre V, Nicouleau M, Defoort-Delhemmes S, Delphin N, Fares-Taie L, Gerber S, Xerri O, Edelson C, Goldenberg A, et al. 2012. Mutations in NMNAT1 cause Leber congenital amaurosis with early-onset severe macular and optic atrophy. Nat Genet 44:975-977.

Perrault I, Rozet JM, Calvas P, Gerber S, Camuzat A, Dollfus H, Chatelin S, Souied E, Ghazi I, Leowski C, Bonnemaison M, Le Paslier D, et al. 1996. Retinal-specific guanylate cyclase gene mutations in Leber's congenital amaurosis. Nat Genet 14:461-464.

Ramprasad VL, Soumittra N, Nancarrow D, Sen P, McKibbin M, Williams GA, Arokiasamy T, Lakshmipathy P, Inglehearn CF, Kumaramanickavel G. 2008. Identification of a novel splice-site mutation in the Lebercilin (LCA5) gene causing Leber congenital amaurosis. Mol Vis 14:481-486.

Sergouniotis PI, Davidson AE, Lenassi E, Devery SR, Moore AT, Webster AR. 2012. Retinal structure, function, and molecular pathologic features in gyrate atrophy. Ophthalmology 119:596-605.

Sergouniotis PI, Davidson AE, Mackay DS, Li Z, Yang X, Plagnol V, Moore AT, Webster AR. 2011. Recessive mutations in KCNJ13, encoding an inwardly rectifying potassium channel subunit, cause leber congenital amaurosis. Am J Hum Genet 89:183-190.

Sohocki MM, Bowne SJ, Sullivan LS, Blackshaw S, Cepko CL, Payne AM, Bhattacharya SS, Khaliq S, Qasim Mehdi S, Birch DG, Harrison WR, Elder FF, et al. 2000. Mutations in a new photoreceptor-pineal gene on $17 \mathrm{p}$ cause Leber congenital amaurosis. Nat Genet 24:79-83.

Swaroop A, Wang QL, Wu W, Cook J, Coats C, Xu S, Chen S, Zack DJ, Sieving PA. 1999. Leber congenital amaurosis caused by a homozygous mutation (R90W) in the homeodomain of the retinal transcription factor CRX: direct evidence for the involvement of CRX in the development of photoreceptor function. Hum Mol Genet 8:299-305.

Thompson DA, Li Y, McHenry CL, Carlson TJ, Ding X, Sieving PA, ApfelstedtSylla E, Gal A. 2001. Mutations in the gene encoding lecithin retinol acyltransferase are associated with early-onset severe retinal dystrophy. Nat Genet 28: 123-124.

Vallespin E, Avila-Fernandez A, Almoguera B, Cantalapiedra D, Garcia-Hoyos M, Riveiro-Alvarez R, Aguirre-Lamban J, Bustamante-Aragones A, Trujillo-Tiebas MJ, Ayuso C. 2010a. Novel human pathological mutations. Gene symbol: LCA5. Disease: Leber Congenital Amaurosis (LCA). Hum Genet 127:118.

Vallespin E, Avila-Fernandez A, Almoguera B, Velez-Monsalve C, Cantalapiedra D, Garcia-Hoyos M, Riveiro-Alvarez R, Aguirre-Lamban J, Bustamante-Aragones A, Trujillo-Tiebas MJ, Ayuso C. 2010b. Novel human pathological mutations. Gene symbol: LCA5. Disease: Leber congenital amaurosis. Hum Genet 127:487.

von Leber T. 1869. Ueber Refinitis pigmentosa und angeborene Amaurose. Archiv fur Ophthalmologie 15:1-25.

Wang H, den Hollander AI, Moayedi Y, Abulimiti A, Li Y, Collin RW, Hoyng CB, Lopez I, Abboud EB, Al-Rajhi AA, Bray M, Lewis RA, et al. 2009. Mutations in SPATA7 cause Leber congenital amaurosis and juvenile retinitis pigmentosa. Am J Hum Genet 84:380-387.

Weleber RG, Michaelides M, Trzupek KM, Stover NB, Stone EM. 2011. The phenotype of Severe Early Childhood Onset Retinal Dystrophy (SECORD) from mutation of RPE65 and differentiation from Leber congenital amaurosis. Invest Ophthalmol Vis Sci 52:292-302.

Zweifel SA, Engelbert M, Laud K, Margolis R, Spaide RF, Freund KB. 2009. Outer retinal tubulation: a novel optical coherence tomography finding. Arch Ophthalmol 127(12):1596-1602. 